\title{
Samambaias e licófitas dos Cerros do Tigre e Palomas, província biogeográfica do Pampa, Estado do Rio Grande do Sul, Brasil
}

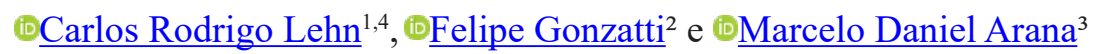

Recebido: 9 julho 2019; aceito: 26 novembro 2019

Como citar: Lehn, C.R., Gonzatti, F. \& Arana, M.D. 2020. Samambaias e licófitas dos Cerros do Tigre e Palomas, província biogeográfica do Pampa, Estado do Rio Grande do Sul, Brasil. Hoehnea 47: e762019. http://dx.doi.org/10.1590/2236-890676/2019.

ABSTRACT - (Ferns and lycophytes from the Cerro do Tigre and Cerro Palomas, Pampa biogeographic province, Rio Grande do Sul State, Brazil) The Pampa represents one of the domains of highest floristic richness in South America, however, especially in relation to the ferns and lycophytes, it still poorly known. This study aims to list the ferns and lycophytes associated with the Tigre and Palomas hills and discuss the floristic aspects that shape the fern and lycophyte flora at the isolated formations (Cerros) of the Pampa domain. The samples were collected and herborized using standard techniques and identified through specific literature. Herbarium specimens were also analyzed in order to complement the floristic list. Species were classified according to their geographical distribution pattern and also considering their life and growth forms. The inventory resulted in 36 species, representing four lycophytes and 32 ferns, distributed in 11 families and 19 genera. Overall, 18 species were exclusive to Cerro do Tigre, five to Cerro Palomas and 13 shared between both areas. Most of the species are widely distributed in South America (41,02\%) and Tropical America (35,89\%), and no endemic species to the pampean domain were identified. The predominant life form was hemicryptophyte, and rosulate was the most represented growth form. In general, the species occurring in the areas presented adaptations to xerophytic conditions. The results showed a high species richness in the studied areas in relation to cited for the whole domain, indicating that the Cerros serve as refuges for the maintenance of ferns and lycophytes in the Pampa region.

Keywords: inselbergs, Pampa domain, pteridophytes, survey

RESUMO - (Samambaias e licófitas dos Cerros do Tigre e Palomas, província biogeográfica do Pampa, Estado do Rio Grande do Sul, Brasil) O Pampa representa um dos domínios fitogeográficos de maior riqueza florística da América do Sul; no entanto, principalmente ao que refere às samambaias e licófitas, ainda é muito pouco conhecido. O presente estudo tem como objetivos listar as espécies de samambaias e licófitas associadas aos cerros do Tigre e Palomas e discutir os aspectos florísticos que modelam a flora de samambaias e licófitas nas formações isoladas (Cerros) do domínio do Pampa. As amostras foram coletadas e herborizadas por meio das técnicas usuais, e identificadas com auxílio de literatura específica. Espécimes de herbários também foram analisados a fim de complementar a lista florística. As espécies foram classificadas quanto seu padrão de distribuição geográfica e quanto sua forma de vida e crescimento. O inventário resultou em 36 espécies, sendo quatro licófitas e 32 samambaias, distribuídas em 11 famílias e 19 gêneros. Ao todo, 18 espécies foram exclusivas ao Cerro do Tigre, cinco ao Cerro Palomas e 13 ocorreram em ambas as áreas. A maior parte das espécies tem ampla distribuição na América do Sul (41,02\%) e América Tropical (35,89\%), e não foram identificadas espécies endêmicas ao domínio pampeano. A forma de vida predominante foi hemicriptófito e a forma de crescimento, o rosulado foi a mais frequente. De forma geral as espécies ocorrentes na área de estudo apresentaram adaptações às condições xerofíticas. Nossos resultados mostraram haver alta riqueza de espécies nas áreas estudadas em relação ao citado para o domínio, e que os Cerros servem como refúgios para manutenção da flora de samambaias e licófitas na região.

Palavras-chave: domínio do Pampa, inselbergs, inventário, pteridófitas

1. Instituto Federal Farroupilha, Campus Panambi, Rua Erechim 860, 98280-000 Panambi, RS, Brasil

2. Universidade de Caxias do Sul, Herbário da Universidade de Caxias do Sul, Museu de Ciências Naturais, Rua Francisco Getúlio Vargas, 1130, 95070-360 Caxias do Sul, RS, Brasil

3. Universidad Nacional de Río Cuarto, Facultad de Ciencias Exactas, Físico-Químicas y Naturales, Departamento de Ciencias Naturales, Ruta 36 km 601, X5804BYA Río Cuarto, Córdoba, Argentina

4. Autor para correspondência: crlehn@gmail.com 


\section{Introdução}

Situado no extremo sul da América do Sul, o Pampa ocupa uma área estimada em mais de 750.000 $\mathrm{km}^{2}$ recobrindo boa parte da Argentina, Uruguai e Sul do Brasil, ocorrendo majoritariamente na metade sul do estado do Rio Grande do Sul e ocupando cerca de $2 \%$ do território nacional brasileiro (IBGE 2012). Como unidade biogeográfica, o Pampa apresenta grandes variações geomorfológicas, pedológicas e fitofisionômicas que originam a alta diversidade de espécies biológicas que ocorrem no domínio (Overbeck et al. 2007, Roesch et al. 2009, Fiaschi \& Pirani 2009), sendo floristicamente caracterizado pela abundância de Poaceae subtropicais dos gêneros Paspalum L., Axonopus P. Beauv., Nassella (Trin.) E. Desv. e Digitaria Haller. Representando uma das mais importantes formações campestres das Américas (Dixon et al. 2014), reúne uma flora com cerca de 4.800 espécies (Andrade et al. 2018), que ocorrem em formações campestres arenosas, campos rochosos e em ambientes florestados como savanas e matas estacionais que se desenvolvem ao longo de ambientes ripários e entorno aos cerros e encostas (Roesch et al. 2009, IBGE 2012).

Os cerros pampeanos constituem morros testemunhos com origem datando do período Jurássico Superior (Oliveira \& Kerber 2009). Tais formações apresentam composição geomorfológica e histórico de formação bastante distintas, que variam desde relictos das formações basálticas da Formação Serra Geral (Cerro Palomas), quanto das formações arenosas da Formação Guará (Cerro do Tigre) (Scherer et al. 2000). Com altitude variando entre 180 e $300 \mathrm{~m}$, estas formações esculpidas pela ação do vento e processos erosivos formam montanhas de formas circulares ou arredondadas, geralmente com um platô desenvolvido (Moura 1991, Verdum et al. 2019), onde abrigam uma flora distinta da verificada na paisagem do entorno, atuando como ilhas de biodiversidade, especialmente de samambaias e licófitas (Arana et al. 2013).

A riqueza de samambaias e licófitas no Brasil é estimada em cerca de 1.300 espécies (Prado et al. 2015, BFG 2018), das quais aproximadamente 400 ocorrem no Rio Grande do Sul (Flora do Brasil 2020). Esta riqueza de espécies é heterogeneamente distribuída entre os domínios que ocorrem no Brasil, sendo que o Pampa constitui o mais pobre floristicamente, com ocorrência reconhecida de somente cinco espécies e inexistência de endemismos (Forzza et al. 2012, Prado $\&$ Hirai 2014). Os recentes avanços no conhecimento acerca da flora do Brasil, ampliados a partir da implantação do projeto Flora do Brasil 2020, tem aumentado os esforços de revisão taxonômica dos grupos e revisão das ocorrências das espécies, no caso das samambaias e licófitas, o número de espécies ocorrentes no Pampa foi triplicado, passando de cinco espécies (Prado \& Sylvestre 2010) para 17 espécies atualmente (BFG 2018).

Mesmo datando mais de 180 anos desde a realização das primeiras coletas botânicas no Pampa, conduzidas por historiadores como Auguste de Saint-Hilaire (início do século XIX), Àrsene Isabelle (1830-1834) e C.A. Lindmann (1892-1894), a riqueza de samambaias e licófitas ocorrentes na região permanece pouco conhecida. Historicamente, tais esforços concentraram-se nas fitofisionomias Floresta Ombrófilas Densa e Mista (ex. Bueno \& Senna 1992, Senna \& Waechter 1997, Schmitt et al. 2006, Santos \& Windisch 2008), Formações Estacionais (ex. Steffens \& Windisch 2007, Lehn et al. 2009, Gonzatti et al. 2014, Padoin et al. 2015) e Formações Pioneiras (Athayde-Filho \& Windisch 2006, Gonzatti et al. 2016), todas pertencentes ao domínio Atlântico.

Atualmente, a expansão das fazendas de pecuária promovendo um incremento das áreas destinada à pastagem (Andrade et al. 2019), juntamente com o cultivo de extensas áreas de florestas de eucalipto e pinus destinadas principalmente à indústria da celulose, estão entre as principais ameaças para a manutenção da integridade dos ecossistemas pampeanos (Buisson et al. 2018), sendo estimado que entre 1985 e 2017 o Pampa tenha sofrido uma redução de $22 \%$ em sua cobertura original (MapBiomas 2018). Outros eventos de alterações das características originais de cobertura e estrutura do solo da região decorrem da pressão do pastejo pelo gado, uso do fogo, a invasão das áreas naturais por espécies exóticas, e o revolvimento do solo para a conversão dos campos naturais por campos de cultivo, principalmente da cultura de soja (Andrade et al. 2015). Na região da Campanha, estima-se que somente $38 \%$ das áreas são remanescentes naturais, e cerca de $15 \%$ das áreas já estejam degradas (Andrade et al. 2015).

Considerando este cenário, o presente estudo tem como objetivos listar as espécies da flora de samambaias e licófitas associadas aos cerros pampeanos do Tigre e Palomas e discutir os aspectos florísticos que modelam a flora de samambaias e licófitas nas formações isoladas (Cerros) do domínio do Pampa. 


\section{Material e métodos}

Áreas de Estudo - O estudo foi realizado nas localidades conhecidas como Cerro do Tigre e Cerro Palomas. O Cerro do Tigre está localizado no município de

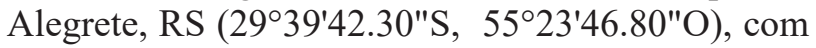
altitude variando entre 130 e $210 \mathrm{~m}$, ocupando uma área próxima de 35 hectares (figura 1a). A área do Cerro do Tigre compreende um maciço de rochas
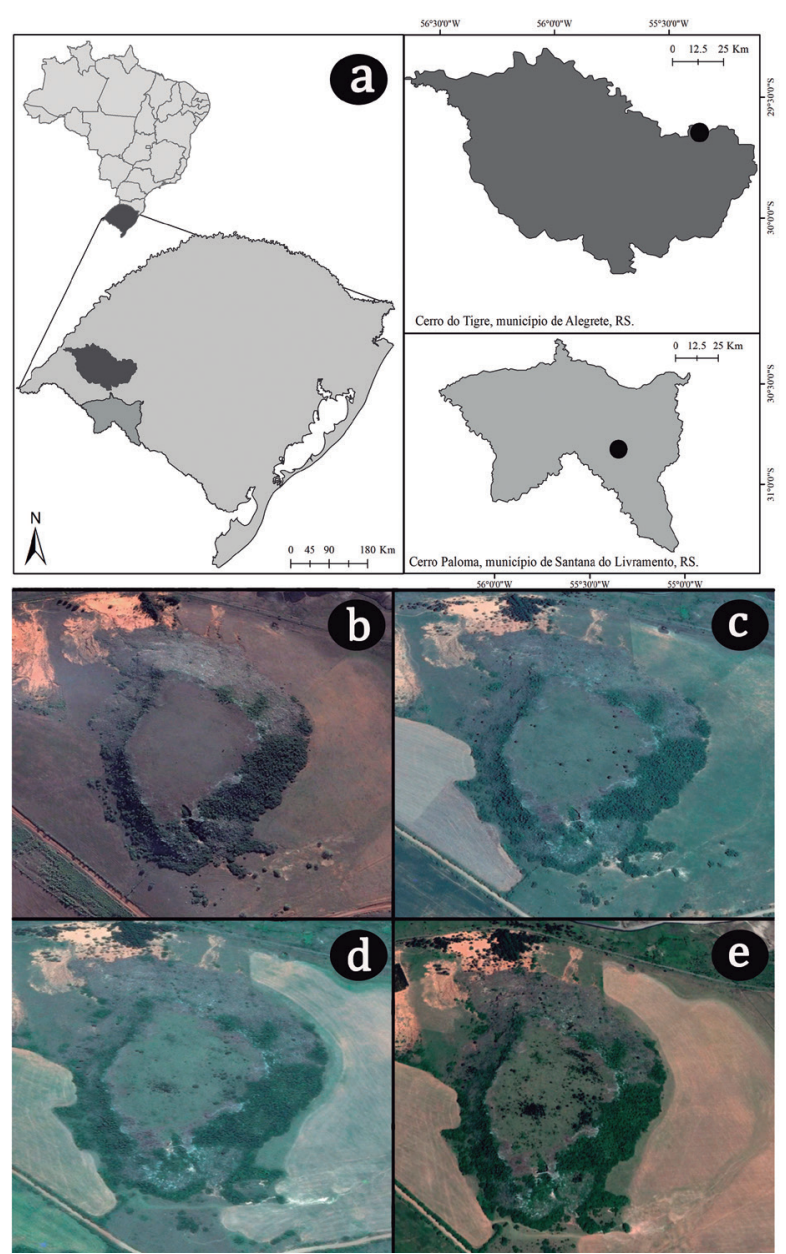

Figura 1. a. Mapa de localização dos Cerros do Tigre e Palomas, província biogeográfica do Pampa, Estado do Rio Grande do Sul, Brasil. b-e. Evolução das alterações paisagísticas no Cerro do Tigre entre os anos de 2004 e 2019, promovidas pela implementação de lavouras de grãos (no entorno) e pelo estabelecimento de uma população de Pinus elliotti L. na área do platô. b. Imagem de satélite do ano de 2004. c. Imagem de satélite do ano de 2013. d. Imagem de satélite do ano de 2016. e. Imagem de satélite de 2019.

Figure 1. a. Location map of Cerros do Tigre and Palomas, Pampean biogeographic province, State of Rio Grande do Sul, Brazil. b-e. evolution of landscape changes in Cerro do Tigre between 2004 and 2019, promoted by the implementation of grain crops (in the neighboring areas) and by the establishment of a population of Pinus elliotti L. in the plateau area. b. Satellite image of the year 2004. c. Satellite image of the year 2013. d. Satellite image of the year 2016. e. 2019 satellite image. areníticas em diferentes estágios de decomposição. As bordas deste Cerro são moldadas por encostas de elevação suave, irregularmente sobrepostas por rochas e taludes onde formam-se pequenas florestas estacionais com predomínio de Syagrus romanzoffiana (Cham.) Glassman (jerivá) como elemento principal do componente arbóreo (figura 2a). Já no topo forma-se um platô recoberto por campos rupestres de caráter herbáceo/arbustivo (figura 2b). Já o Cerro Palomas, situa-se no município de Santana do Livramento, RS (3050'5.17"S, 55²5'52.85"O), com altitude entre 200 e $300 \mathrm{~m}$, em uma área de cerca de 10 hectares. A matriz rochosa que forma o Cerro Palomas é de origem basáltica, o que faz com que as faces do cerro sejam mais abruptas, formando taludes retos e altos. No ápice, há a formação de um platô onde desenvolve-se campo rupestre herbáceo/ arbustivo (figura 2c). No platô, baixadas húmidas são evidenciadas em áreas onde a ocorre a deposição da água, já na base, desenvolvem-se pequenos capões de matas estacionais que não atingem o topo do cerro. Ambas as áreas, tem sofrido uma intensa descaracterização das paisagens naturais do seu entorno, devido à conversão dos campos naturais em campos de cultivo agrícola (figura 1b-e).

De acordo com a classificação climática apresentada em Peel et al. (2007), o clima da região é definido como temperado, sem a existência de estação seca e com verões quentes, com temperatura média superior a $22{ }^{\circ} \mathrm{C}$, inserido no subtipo $C f a$.

Coletas, Herborização e Identificação - Foram realizadas dez expedições de amostragem, distribuídas entre os anos de 2003 e 2018. Em cada área, com o emprego do método do caminhamento (Filgueiras et al. 1994), foram realizadas buscas utilizando-se principalmente trilhas pré-existentes, nos pontos de entorno das bases, nas encostas e nos topos dos cerros, buscando-se contemplar os diferentes microambientes existentes.

As coletas de material testemunho foram realizadas em acordo com técnicas usuais para samambaias e licófitas descritas em Windisch (1992). Após o devido processamento, os materiais coletados foram incorporados nos herbários HUCS e PACA. Revisões adicionais foram realizadas nos herbários HUCS, PACA, HVAT e ICN para consulta e confirmação de ocorrências de espécies não amostradas pelos autores (acrônimos conforme, Thiers 2019). Todas as amostras citadas para as respectivas localidades estudas foram analisadas. A 


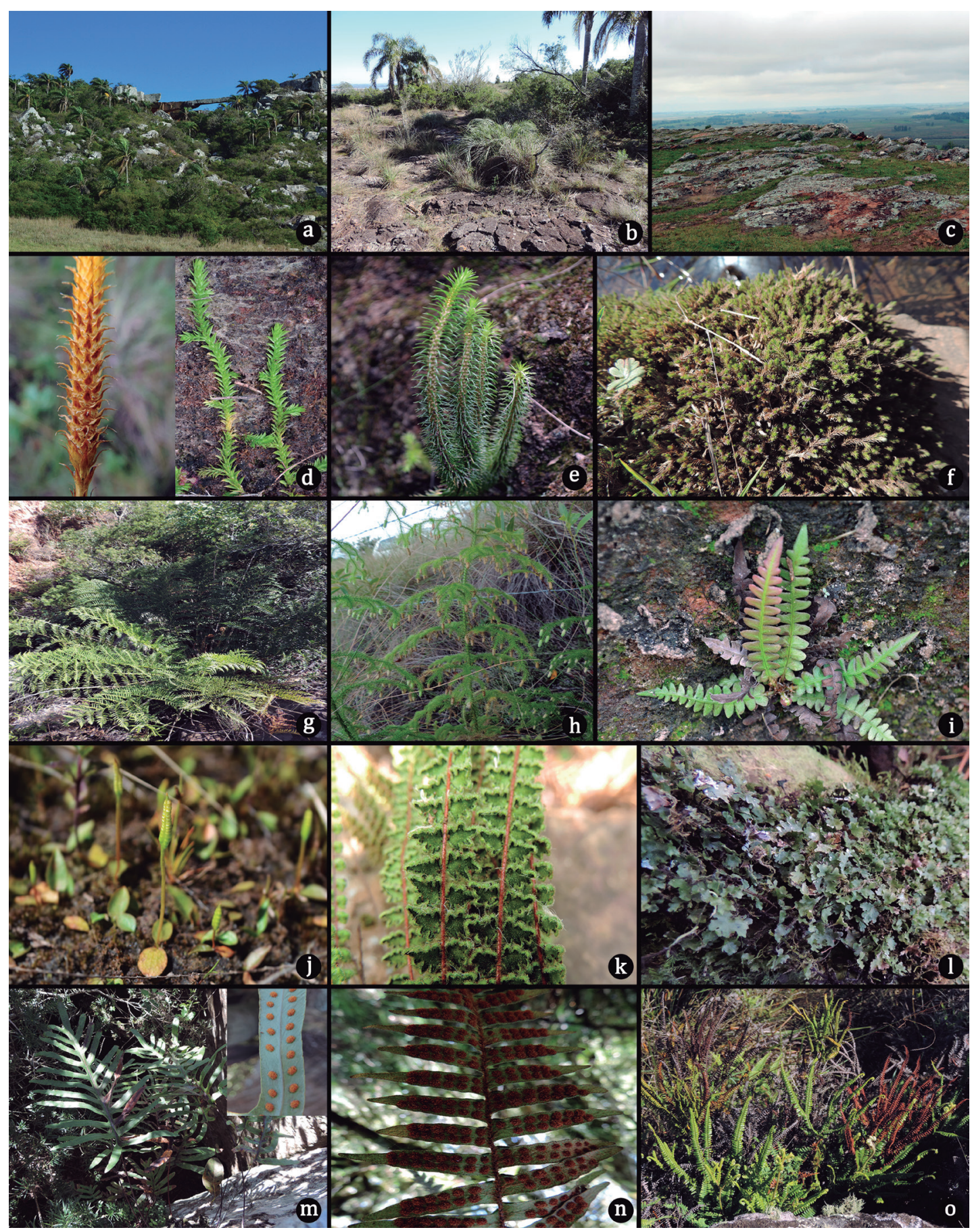

Figura 2 - Samambaias e licófitas dos Cerros Palomas e do Tigre, província biogeográfica do Pampa, Estado do Rio Grande do Sul, Brasil. a. Vista Geral do Cerro do Tigre. b. Platô, Cerro do Tigre. c. Platô, Cerro Palomas. d. Pseudolycopodiella caroliniana (L.) Holub. e. Phlegmariurus reflexus (Lam.) B. Øllg. f. Selaginella sellowii Hieron. g. Cyathea atrovirens (Langsd. \& Fisch.) Domin. h. Palhinhaea cernua (L.) Franco \& Vasc. i. Blechnum polypodioides Raddi. j. Ophioglossum nudicaule L.f. k. Cheilanthes micropteris Sw. 1. Didymoglossum hymenoides (Hedw.) Copel. m. Phlebodium pseudoaureum (Cav.) Lellinger. n. Pleopeltis hirsutissima (Raddi) de la Sota e o. Blechnum asplenioides Sw.

Figure 2 - Ferns and lycophytes from Cerros Palomas and Tigre, Pampean biogeographic province, State of Rio Grande do Sul, Brazil.a. General view of Cerro do Tigre. b. Plateau, Cerro do Tigre. c. Plateau, Cerro Palomas. d. Pseudolycopodiella caroliniana (L.) Holub. e. Phlegmariurus reflexus (Lam.) B. Øllg. f. Selaginella sellowii Hieron. g. Cyathea atrovirens (Langsd. \& Fisch.) Domin. h. Palhinhaea cernua (L.) Franco \& Vasc. i. Blechnum polypodioides Raddi. j. Ophioglossum nudicaule L.f. k. Cheilanthes micropteris Sw. 1. Didymoglossum hymenoides (Hedw.) Copel. m. Phlebodium pseudoaureum (Cav.) Lellinger. n. Pleopeltis hirsutissima (Raddi) de la Sota e o. Blechnum asplenioides Sw. 
circunscrição das famílias de samambaias e licófitas segue a delimitação proposta em PPG I (2016), e a redação nomenclatural segue o International Plant Names Index (IPNI).

Padrão de distribuição geográfica - O padrão de distribuição geográfica das espécies é apresentado tendo como base informações disponíveis na literatura relativa às espécies observadas nas áreas de estudo (Dittrich et al. 2015, Gasper \& Salino 2016, Arana et al. 2017), sendo estes: AS - espécies com ocorrência ampla na América do Sul; AT - espécies com ocorrência ampla na América Tropical; EBV - espécie endêmica do Brasil e áreas vizinhas; PA - espécie com distribuição pantropical (trópicos e subtrópicos do Novo e Velho Mundo).

Formas de Vida/Crescimento - As espécies inventariadas foram classificadas de acordo com a sua forma de vida/crescimento, seguindo critérios apresentados em Mueller-Dombois \& Ellenberg (1974) e Senna \& Waechter (1997). Formas de vida: Hemicriptófita (HC) - apresenta gema de perenização situada ao nível do solo ou ligeiramente acima deste; Fanerófita (FA) - apresenta gema de perenização, geralmente, acima de $50 \mathrm{~cm}$ de altura em relação ao solo; Geófita (GE) - apresenta gema de perenização abaixo da superfície do solo; Caméfita (CAM) apresenta gema de perenização acima do nível do solo, dificilmente ultrapassando $50 \mathrm{~cm}$ de altura; Terófita (TER) - se caracterizam por desenvolver-se anualmente, atingindo a senescência após a liberação dos esporos. Formas de crescimento: Reptante (REP) característica marcante de espécies com rizomas longos e horizontais; Rosulada (ROS) - apresenta frondes com disposição helicoidal, concentradas na porção terminal do rizoma; Rizomatosa (RIZ) - apresentam rizoma subterrâneo e disposição dorsiventral.

\section{Resultados}

Do esforço amostral e das revisões dos acervos, foram avaliados 55 espécimes pertencentes a 36 espécies (quatro licófitas e 32 samambaias) (tabela 1 , figura 2). As samambaias estão representadas por 11 famílias e 19 gêneros, enquanto que para as licófitas foram registradas duas famílias (Lycopodiaceae e Selaginellaceae) e quatro gêneros. O Cerro do Tigre reuniu o maior número de espécies (31 spp.), das quais 18 apresentaram ocorrência exclusiva. Para o Cerro Palomas, foi registrada a ocorrência de 18 espécies, das quais seis foram verificadas somente para esta área. Ambas as áreas compartilham a ocorrência de 13 espécies.

Considerando a soma das duas áreas, Pteridaceae e Blechnaceae apresentaram as maiores riquezas, representadas respectivamente por oito e sete espécies. Juntamente com Polypodiaceae, reúnem cerca de $60 \%$ das espécies inventariadas. Blechnum L. foi o gênero de ocorrência mais expressiva, representado por seis espécies.

As espécies observadas nas áreas de estudo ocorrem amplamente na América do Sul (41,02\%) e América Tropical (35,89\%) (figura 3). A forma de vida hemicriptófita (32 spp.) e a forma de crescimento rosulada (17 spp.) predominam entre as espécies registradas (figura 4).

\section{Discussão}

A flora de samambaias e licófitas encontradas nos dois cerros estudados apresentam riqueza de espécies superior ao indicado pelo BFG (2018) para o domínio. Em uma compilação pioneira para o Pampa do Rio Grande do Sul, Argentina e Uruguai, Andrade et al. (2018) reportam a ocorrência de 198 espécies, sendo 16 licófitas e 182 samambaias. Segundo os autores, dentre as espécies de licófitas ocorrentes no domínio do Pampa, apenas sete são compartilhadas entre todas as formações dos três países (Argentina, Brasil e Uruguai), sendo que quatro são exclusivas às formações pampeanas do Rio Grande do Sul. Já em relação às samambaias, este compartilhamento ocorre entre 87 espécies, enquanto que 27 são de ocorrência restrita ao Pampa brasileiro. Estes dados são similares aos encontrados para a flora de samambaias e licófitas do Uruguai, que somam 115 espécies (Brussa \& Grela 2005). O Pampa como um todo, apresenta riqueza de espécies cerca de $80 \%$ menor que as formações tropicais da Mata Atlântica (Prado et al. 2015). A baixa riqueza de espécies no Pampa está relacionada a fatores como gradientes latitudinal e continental de diversidade, que reduzem a riqueza florística no sentido Norte-Sul e Leste-Oeste em direção às formações pampeanas em função das influências fitogeográficas, climáticas e de processos migratórios de vegetação (Gonzatti et al. 2016, Machado et al. 2016).

Segundo Sehnem (1977), as maiores riquezas florísticas de samambaias e licófitas no Rio Grande do Sul concentram-se nas florestas ombrófilas e pluviais da porção norte do estado devido ao caráter higrófito das espécies, e que nas áreas de campo (Pampa) ocorrem 


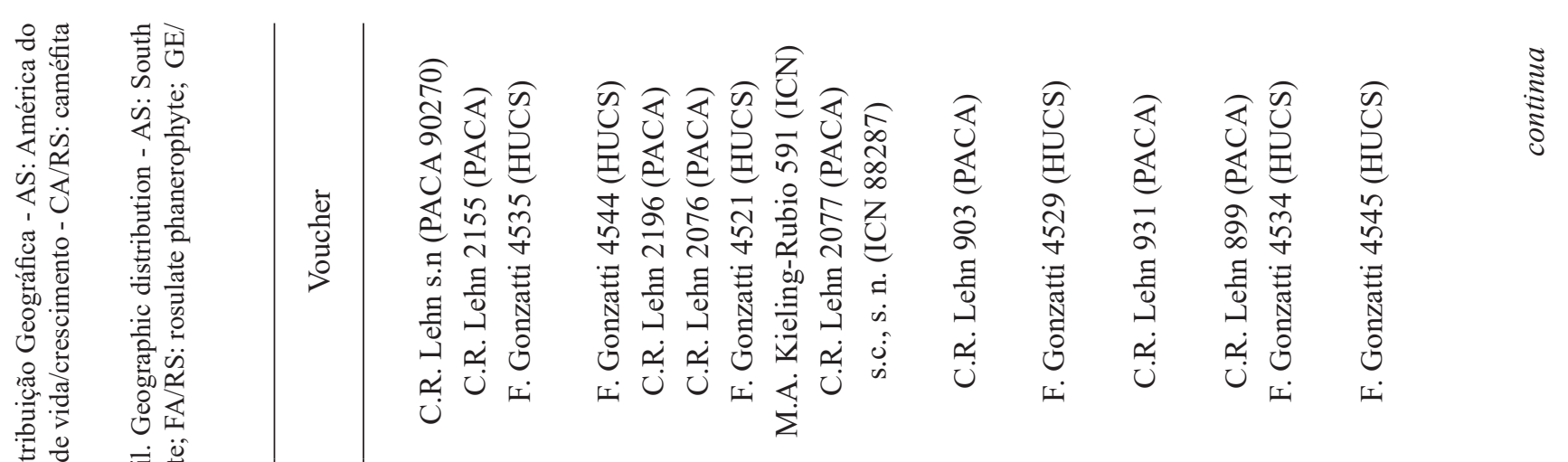

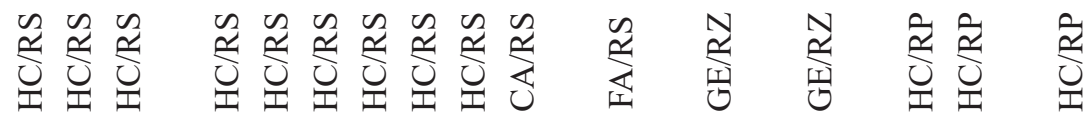

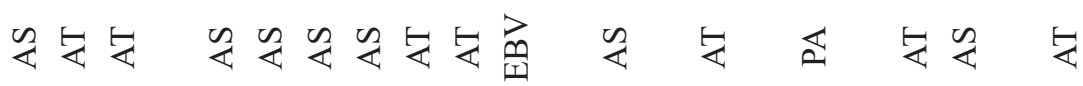

\& $8 \cdot 0$.

远.

w

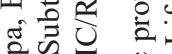

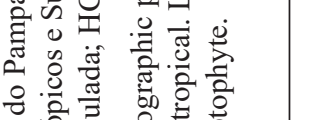

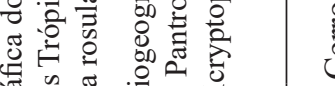

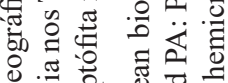

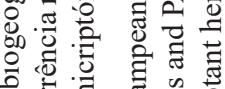

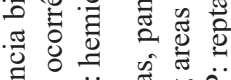

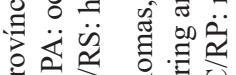

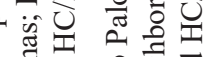

零

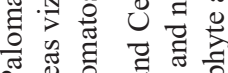

2.

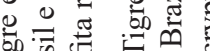

跑总。용

8 ○ी

ธ ชิ

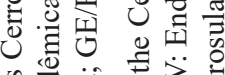

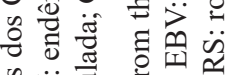

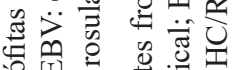

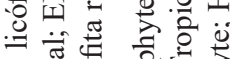

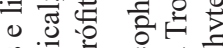

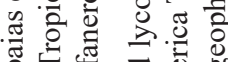

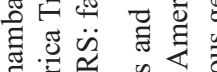

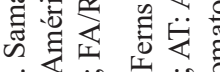

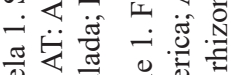

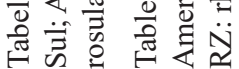

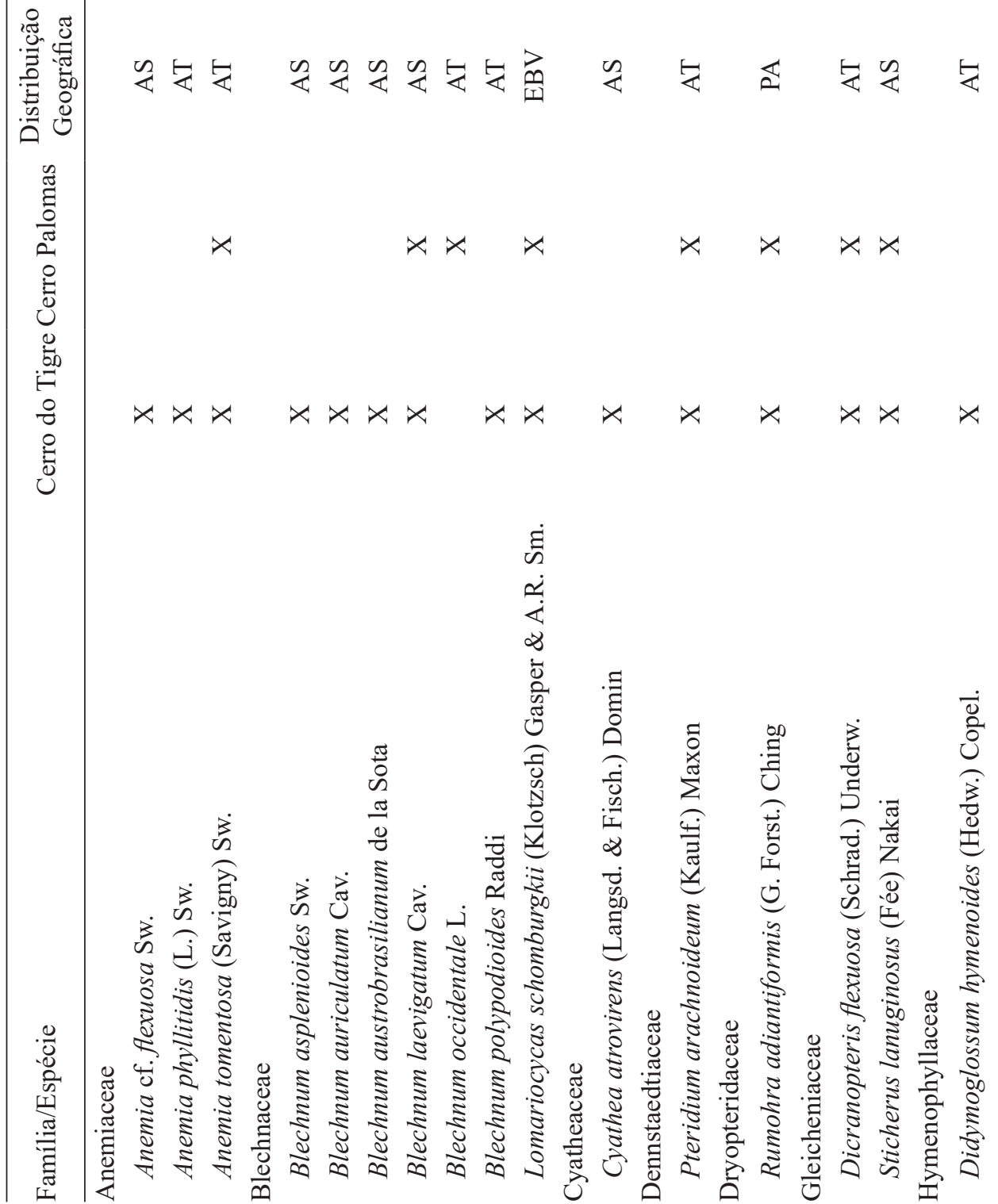




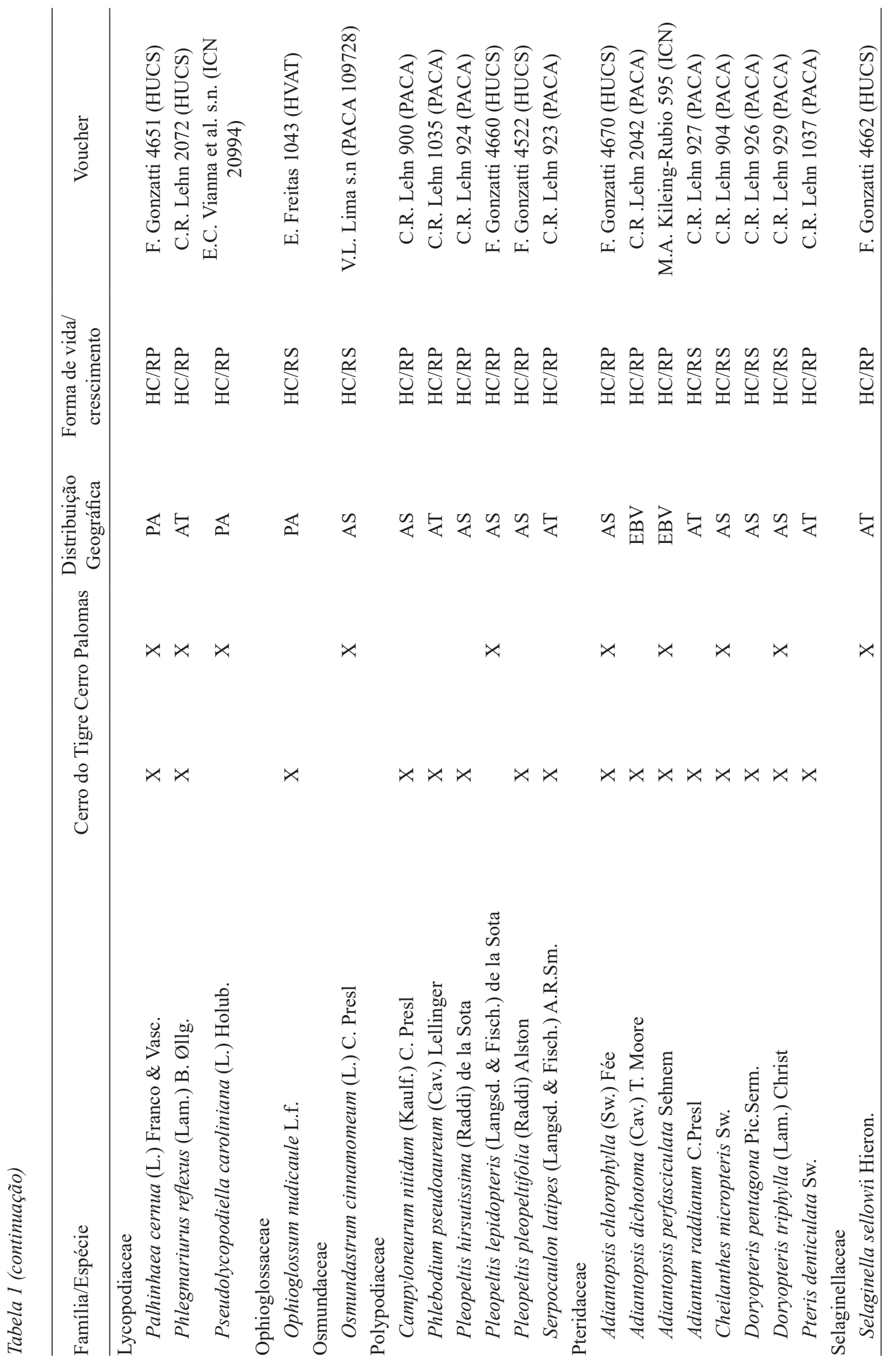




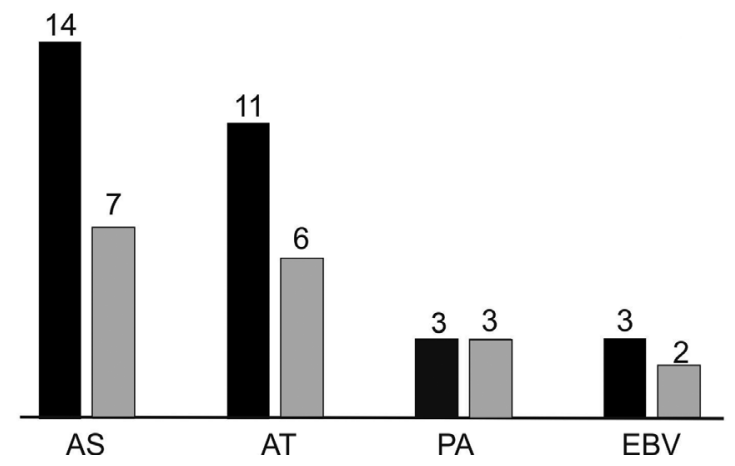

Figura 3. Número de espécies de samambaias e licófitas registradas no Cerro do Tigre e Cerro Palomas, província biogeográfica do Pampa, Estado do Rio Grande do Sul, Brasil, classificadas de acordo com sua distribuição geográfica - AS: América do Sul. AT: América Tropical. EBV: Endêmica do Brasil e áreas vizinhas e PA: Pantropical. Cerro do Tigre, $\square$ Cerro Palomas.

Figure 3. Number of species of ferns and lycophytes registered in Cerro do Tigre and Cerro Palomas, Pampean biogeographic province, State of Rio Grande do Sul, Brazil, classified according to their geographic distribution - AS: South America. AT: America Tropical. EBV: Endemic to Brazil and neighboring areas and PA: Pantropical. $\square$ Cerro do Tigre, $\square$ Cerro Palomas.

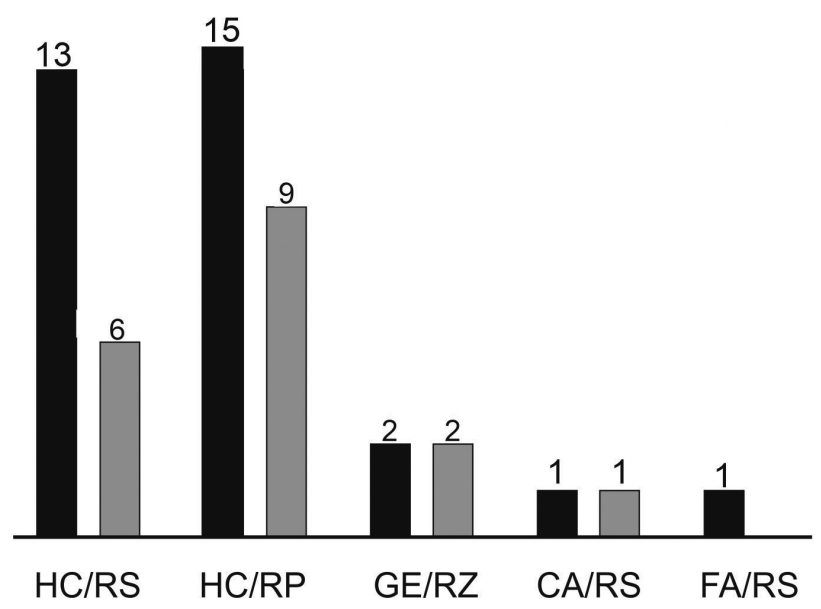

Figura 4. Número de espécies de samambaias e licófitas observadas no Cerro do Tigre e no Cerro Palomas, província biogeográfica do Pampa, Estado do Rio Grande do Sul, Brasil, classificadas de acordo com suas respectivas formas de vida/crescimento - HC/RS: hemicriptófita rosulada. HC/RP: hemicriptófita reptante. GE/RZ: geófita rizomatosa e CA/RS: caméfita rosulada. FA/RS: fanerófita rosulada. Cerro do Tigre, - Cerro Palomas.

Figure 4. Number of species of ferns and lycophytes observed in Cerro do Tigre and Cerro Palomas, Pampean biogeographic province, State of Rio Grande do Sul, Brazil, classified according to their respective life/growth forms - HC/RS: rosulate hemicryptophyte. HC/RP: reptant hemicryptophyte. GE/RZ: rhizomatous geophyte and CA/RS: rosulate camefite. FA/RS: rosulate phanerophyte. Cerro do Tigre, Cerro Palomas. somente espécies de ampla distribuição geográfica ou de caráter ecológico pioneiro que migraram das regiões setentrionais para o Sul e encontram no Pampa seu limite de distribuição. Este efeito foi observado com Lomariocycas schomburgkii que apresenta ampla distribuição ao longo das formações montanhosas das formações atlânticas desde a Bahia até o Rio Grande do Sul (Dittrich et al. 2018), e que ocorreu esporadicamente no Cerro Palomas.

No entanto, alguns ambientes presentes no domínio do Pampa têm papel importante para a ocorrência de samambaias e licófitas devido a formação de nichos ecológicos que propiciam o desenvolvimento de espécies mais dependentes de umidade ou sombreamento. Este é o caso das florestas estacionais ripárias, que percorrem os cursos hídricos e conectam diferentes fitofisionomias e que possivelmente atuam como corredores para a dispersão de espécies. Na área de estudo, o rio Ibicuí, por exemplo, que através da conexão com o Rio Uruguai e Rio Jaguari forma um corredor entre as formações da Mata Atlântica do Alto Uruguai com as formações da calha central do Estado (região de Santa Maria).

A presença de formações isoladas como os Cerros Paloma e do Tigre, que pela diferenciação geológica, altitudinal e fitofisionômica da matriz do entorno atuam como inselbergs, formando hábitat para a ocorrência de espécies com nichos específicos além de oferecer condições para processos de especiação (Porembski 2007). No caso do Cerro do Tigre, a presença de encostas com degraus de diferentes alturas permite o desenvolvimento de nichos diferenciados para grupos florísticos distintos, como fendas de rochas sombreadas ou expostas, bancos de areia deposicionais, afloramentos e costões rochosos, campos abertos ou florestas de encosta. Nestas encostas, pequenos capões de mata se desenvolvem formando ambientes para espécies de caráter florestal como Anemia phyllitidis (figura 5e), Blechnum polypodioides (figura 2i), Adiantum raddianum (figura 5f) e Adiantopsis perfasciculata (figura 5c). Em angiospermas, novas ocorrências e espécies novas têm sido descritas com frequência para estas áreas, em vários grupos taxonômicos como Asteraceae (Mondin 2007), Bromeliaceae (Büneker et al. 2015) e Poaceae (Welker \& Longhi-Wagner 2012).

No que tange às samambaias e licófitas, estes cerros atuam como refúgios para espécies que apresentam especificidade de nicho, substrato ou até 


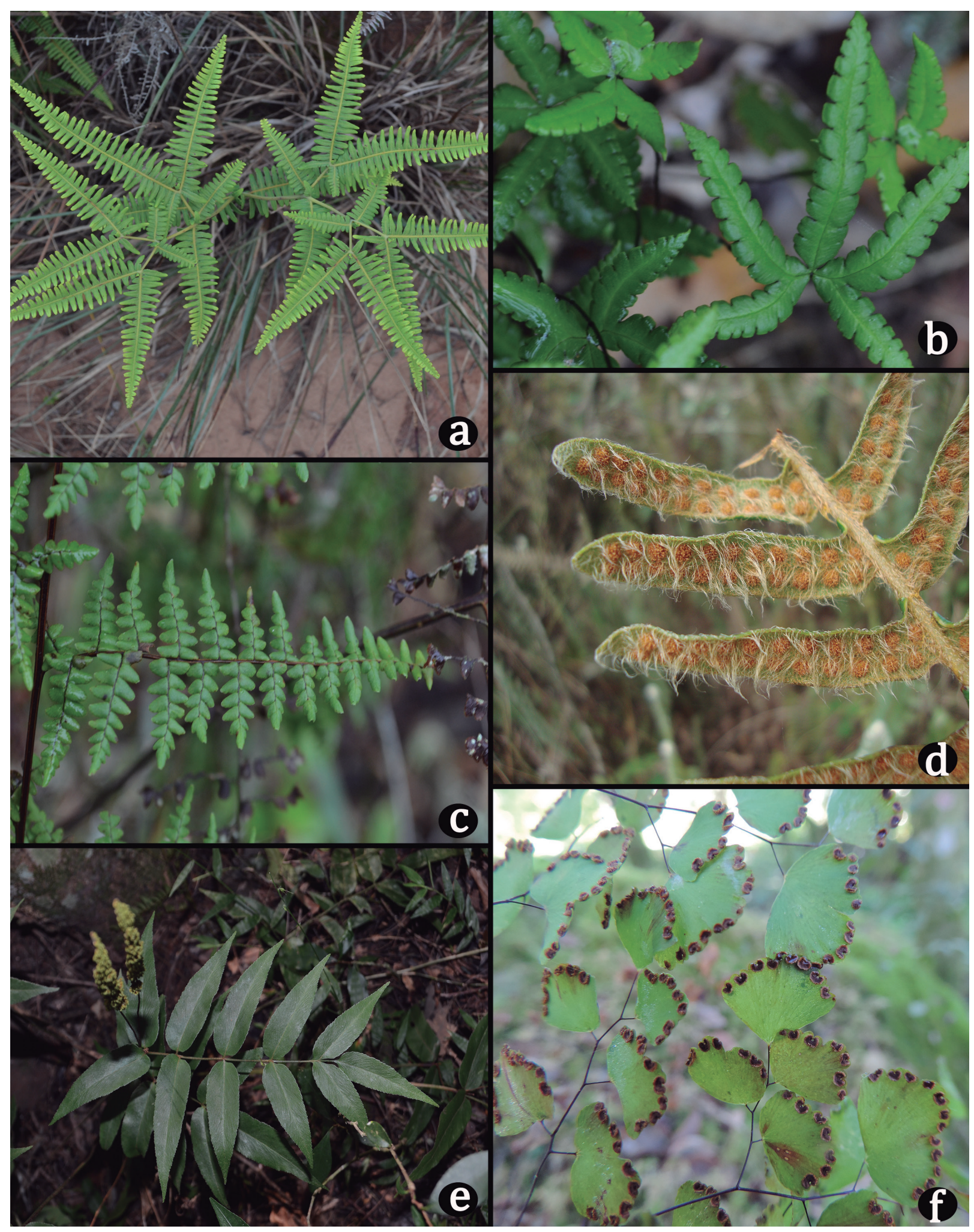

Figura 5 - Samambaias e licófitas dos Cerros Palomas e do Tigre, província biogeográfica do Pampa, Estado do Rio Grande do Sul, Brasil. a. Dicranopteris flexuosa (Schrad.) Underw. b. Doryopteris triphylla (Lam.) Christ. c. Adiantopsis perfasciculata Sehnem. d. Pleopeltis lepidopteris (Langsd. \& Fisch.) de la Sota. e. Anemia phyllitidis (L.) Sw. f. Adiantum raddianum C.Presl.

Figure 5 - Ferns and lycophytes from Cerros Palomas and Tigre, Pampean biogeographic province, State of Rio Grande do Sul, Brazil. a. Dicranopteris flexuosa (Schrad.) Underw. b. Doryopteris triphylla (Lam.) Christ. c. Adiantopsis perfasciculata Sehnem. d. Pleopeltis lepidopteris (Langsd. \& Fisch.) de la Sota. e. Anemia phyllitidis (L.) Sw. f. Adiantum raddianum C.Presl. 
especialização como poiquiloidria (Porembski \& Barthlott 2000). Elementos florísticos tropicais como Cyathea atrovirens (figura 2g) e Didymoglossum hymenoides (figura 21), encontram nestes ambientes sombreados e com maior retenção de umidade do que a da paisagem do entorno, permitindo o seu desenvolvimento e consequente aumento da extensão de ocorrência, fazendo com que consigam adentrar até o Uruguai (Brussa \& Grela 2005). Já espécies de caráter xerofítico como Selaginella sellowii (figura 2f), Cheilanthes micropteris (figura 2k), Pleopeltis hirsutissima (figura 2n), Pleopeltis lepidopteris (figura 5d), Doryopteris triphylla (figura 5b), Sticherus lanuginosus (figura 2o), Dicranopteris flexuosa (figura 5a) e Phlebodium pseudoaureum (figura $2 \mathrm{~m}$ ) são frequentes em afloramentos rochosos ou epífitos em capões de mata.

Dentre os principais elementos florísticos encontrados, Pteridaceae apresenta elevada riqueza no território brasileiro (Prado et al. 2015), estando entre as famílias de maior representatividade em diversos estudos realizados no sul do Brasil (p. ex. Steffens \& Windisch 2007, Lehn et al. 2009, Lautert et al. 2015, Lehn et al. 2018) e em regiões limítrofes da Argentina (p. ex. Márquez et al. 2006, Torres et al. 2013). Embora seja uma família com diversas linhagens adaptadas a ambientes úmidos, uma das características verificadas para alguns representantes da família Pteridaceae é a tolerância a períodos secos, entre estes os gêneros Pityrogramma Link (Tryon \& Tryon 1982), Cheilanthes Sw. (Ponce \& Scataglini 2018) e ainda no caso de Doryopteris J. Sm., sendo frequente a ocorrência associada a ambientes rupestres (Tryon \& Tryon 1982), o que seria um dos fatores que pode explicar a elevada representatividade da família nas duas áreas de estudo.

Embora haja o predomínio de ambientes secos e espécies adaptadas a eles, no topo dos cerros geralmente formam-se platôs que podem apresentar pequenos acúmulos de água sazonais, ou então formar pequenos campos úmidos. Estas condições podem ocorrer na base destas formações, devido ao escoamento da água dos cerros, permitindo a ocorrência de espécies relacionadas a terrenos úmidos, como Ophioglossum nudicaule (figura 2j), Pseudolycopodiella caroliniana (figura 2d) e Palhinhaea cernua (figura 2h).

Com exceção de Pleopeltis lepidopteris, que no Rio Grande do Sul apresenta ampla ocorrência nos terrenos arenosos da região litorânea (Sehnem 1970), espécies de Polypodiaceae frequentemente estão relacionadas ao hábito epifítico. No entanto, nas áreas de estudo, representantes da família foram observados ocupando fendas entre as rochas, com ou sem depósitos húmicos. Entre estas espécies, pode-se observar uma série de características rizomas suculentos, frondes cobertas por escamas (Kluge \& Kessler 2010) e ainda comportamento poiquilohídrico, que as permitem sobreviver em ambientes com oscilações nos níveis de umidade, como fendas entre as rochas e ambientes expostos.

Embora Blechnum L. seja um gênero encontrado preferencialmente em ambientes montanhosos e úmidos, sua ocorrência associada a terrenos rochosos e inclinados, como se caracterizam as áreas de estudo, tem sido reconhecida por diferentes autores (p. ex. Tryon \& Tryon 1982, Dittrich et al. 2015). A exceção de Lomariocycas shomburgkii, as demais espécies pertencentes à família Blechnaceae verificadas no presente estudo integram o subgrupo Blechnum occidentale (Dittrich et al. 2015), que reúne espécies que se caracterizam por apresentar ampla distribuição geográfica e com marcada plasticidade ecológica, ocorrendo tanto em ambientes florestais, na faixa de borda e interior dos fragmentos, bem como em áreas abertas e com características rupestres.

Em geral, as espécies observadas nas áreas de estudo apresentam ampla distribuição na região neotropical, com estreitas relações biogeográficas com os sistemas pampeanos serranos do Uruguai e Argentina, formando parte de uma biota ancestral distribuída principalmente pelo Sul do Brasil, Uruguai e os sistemas serranos da Argentina central (Arana et al. 2013). As conexões florísticas entre as serras pampeanas argentinas e as do Sul do Brasil, formam um padrão ancestral demonstrado para as Asteraceae (Crisci et al. 2001), samambaias como Anemia tomentosa, Blechnum auriculatum e Doryopteris triphylla, reforçando a existência do padrão denominado Arco Peripampásico Serrano (Arana et al. 2013). Este padrão é compartilhado também por outros grupos de organismos, como os opiliões Ceratomontia centralis Maury \& Roig Alsina, 1985 e C. argentina Canals, 1939, encontrados nas Serras de Buenos Aires e também no Uruguai e no sul do Brasil, bem como para os escorpiões do gênero Bothriurus Peters, 1861, em especial os grupos flavidus e prospicuus (Acosta 2002). A distribuição destes organismos não é contínua no domínio do Pampa, sendo que se restringe às formações serranas, que geralmente possuem heterogeneidade de ambientes e microclimas, interrompidos por planícies marcadas pela homogeneidade microambiental e pelos 
períodos sazonais de disponibilidade de água que se constituem um impedimento para o estabelecimento exitoso de licófitas e samambaias (Arana et al. 2013).

A forma de vida hemicriptófita é a mais comum entre as espécies observadas nas áreas de estudo. De uma forma geral, a predominância de hemicriptófitas se mostra como um padrão frequente para estudos envolvendo samambaias e licófitas no sul do Brasil (p. ex. Farias et al. 2014, Rossetto \& Vieira 2013, Moraes et al. 2018, Lehn et al. 2018, Moraes \& Lehn 2019). Em se tratando de ambientes sujeitos à períodos de irregularidade no suporte hídrico, como é o caso de áreas com terrenos rupestres, a localização da gema de perenização nas hemicriptófitas pode contribuir para a sua manutenção durante períodos desfavoráveis (Kornás 1977, 1985). Dessa forma, ao que tudo indica, a evolução dos ambientes nos cerros estudados se mostrou favorável à ocupação pelas hemicriptófitas. Adicionalmente, a disposição rosulada das frondes direciona a água da chuva para a região de crescimento, facilitando ainda a obtenção de nutrientes a partir da decomposição da serapilheira retida na coroa de frondes (Zona \& Christenhusz 2015), nutrientes estes que dadas as microcondições ambientais se mostram pouco acessíveis a estes indivíduos.

A ausência do componente epifítico é uma das características marcantes na flora de samambaias e licófitas associadas aos cerros estudados. Mesmo sendo verificada a ocorrência de indivíduos de porte arbóreo no cinturão basal das áreas de estudo, especialmente no Cerro do Tigre, samambaias e licófitas epífitas não foram encontradas nestas áreas. Cercados por uma matriz caracterizada por uma paisagem aberta, dominada principalmente por vegetação herbácea, a baixa densidade de espécies arbóreas junto aos cerros, associada a ocorrência frequente de ventos e incidência luminosa plena são fatores limitantes para a ocupação do ambiente epifítico.

Embora o domínio pampeano pareça representar uma unidade fitogeográfica bastante homogênea, variações geomorfológicas, climáticas e paisagísticas (Verdum et al. 2019) tornam o Pampa um mosaico de condições fitoecológicas distintas. Muitas destas áreas apresentam, aparentemente, nichos ecológicos menos favoráveis ao desenvolvimento de muitas espécies de samambaias e licófitas. No entanto, formações como as dos cerros aqui estudados, surpreendem pela elevada riqueza de espécies para este grupo florístico, frente ao que é apresentado na literatura atual. Potencialmente outras fitofisionomias, ainda pouco amostradas e sem estudos florísticos específicos, como matas ciliares ou coxilhas recobertas por vegetação arbustivo-arbórea, apresentem também alta riqueza de espécies. Neste sentido, frente a acelerada expansão agrícola, como demonstrado no Cerro do Tigre (figura $1 \mathrm{~b}$-e), que vem convertendo a paisagem pampeana em áreas produtivas de grãos e de madeira, evidencia-se a necessidade de ampliar o conhecimento da riqueza e da ecologia das samambaias e licófitas do domínio pampeano como um todo.

\section{Agradecimentos}

CRL agradece ao Instituto Federal Farroupilhacampus Panambi e à Universidade do Vale do Rio dos Sinos pelo suporte logístico para realização das coletas no Cerro do Tigre; FG agradece a Universidade de Caxias do Sul, pelo suporte para realização das coletas em ambas as áreas de estudo.

\section{Literatura citada}

Acosta, L.E. 2002. Patrones zoogeográficos de los opiliones argentinos (Arachnida: Opiliones). Revista Ibérica de Aracnología 6: 69-84.

Andrade, B.O., Koch, C., Boldrini, I.I., Vélez-Martind, E., Hasenacke, H., Hermannb, J.N., Kollmannb, J., Pillar, V.P., Overbeck, G.E. 2015. Grassland degradation and restoration: a conceptual framework of stages and thresholds illustrated by southern Brazilian grasslands. Natureza \& Conservação 13: 95-104.

Andrade, B.O., Marchesi, E., Burkart, S., Setubal, R. B., Lezama, F., Perelman, S., Schneider, A. A., Trevisan, R., Overbeck, G.E. \& Boldrini, I.I. 2018. Vascular plant species richness and distribution in the Río de La Plata grasslands. Botanical Journal of the Linnean Society 188: 250-256.

Andrade, B.O., Bonilha, C.L., Overbeck, G.E., Vélez-Martin, E., Rolim, R.G., Bordignon, S.A.L., Schneider, A.A., Vogel, E.C., Dióber, B.L., Garcia, E.N, Santos, E.D., Torchelsen, F.P., Vieira, M.S., Silva-Filho, P.J.S., Ferreira, P.M.A., Trevisan, R., Hollas, R., Campestrini, S., Pillar, V. \& Boldrini, I.I. 2019. Classification of South Brazilian grasslands: Implications for conservation. Applied Vegetation Science 22: 168-184.

Arana, M.D., Ponce, M., Morrone, J.J. \& Oggero, A.J. 2013. Patrones biogeográficos de los helechos de las Sierras de Córdoba (Argentina) y sus implicancias en la conservación. Gayana Botánica 70: 357-376.

Arana, M.D., Gonzalez, H.A., Bonifacino, M. \& Brussa, C.A. 2017. A revision of Lycopodiaceae from Uruguay. International Journal of Advanced Botany 3: 24-39.

Athayde-Filho, F.P. \& Windisch, P.G. 2006. Florística e aspectos ecológicos das pteridófitas em uma floresta de Restinga no estado do Rio Grande do Sul, Brasil. Iheringia, série botânica 61: 63-71. 
BFG. 2018. Brazilian Flora 2020: Innovation and collaboration to meet Target 1 of the Global Strategy for Plant Conservation. Rodriguésia 69: 1513-1527.

Brussa C.A. \& Grela, I. 2005. Los helechos como integrantes del bosque indígena: revisión taxonómica de pteridophyta de la flora uruguaya. In: Seminario Compartiendo conocimientos sobre el monte indígena. Montevideo, Movimiento Mundial por los Bosques Tropicales.

Bueno, R.M. \& Senna, R.M. 1992. Pteridófitas do Parque Nacional dos Aparados da Serra. I. Região do Paradouro. Caderno de Pesquisa, série botânica 4: 5-12.

Buisson, E., Le Stradic, S., Silveira, F.A.O., Durigan, G. Overbeck, G.E., Fidelis, A., Fernandes, G.W., Bond, W.J., Hermann, J.M., Mahy, G., Alvarado, S.T., Zaloumis, N.P., \& Veldman, J. W. 2018. Resilience and restoration of tropical and subtropical grasslands, savannas, and grassy woodlands. Biological Reviews 94: $1-20$

Büneker, H.M., Pontes, R.C. \& Witeck-Neto, L. 2015. Novos registros em Tillandsia L. (Bromeliaceae, Tillandsioideae) para o Rio Grande do Sul, Brasil. Rodriguésia 66: 493-498.

Crisci, J.V., Freire, S.E., Sancho, G. \& Katinas, L. 2001. Historical biogeography of the Asteraceae from Tandilia and Ventania Mountain ranges. Caldasia 23: 21-41.

Dittrich, V.A.O., Salino, A. \& Monteiro, R. 2015. The Blechnum occidentale (Blechnaceae, Polypodiopsida) species group in southern and southeastern Brazil. Phytotaxa 231: 201-229.

Dittrich, V.A.O., Salino, A., Monteiro, R. \& Gasper, A.L. 2018. The fern genera Lomaria, Lomariocycas and Parablechnum (Blechnaceae, Polypodiopsida) in southern and southeastern Brazil. Phytotaxa 362: 245-262.

Dixon, A.P., Faber-Langendoen, D., Josse, C., Morrison, J. \& Loucks, C.J. 2014. Distribution mapping of world grassland types. Journal of Biogeography 41: 2003-2019.

Farias, A.P.S., Klein, C.L., Garlet, T.M.B. \& Essi, L. 2014. Pteridoflora da Universidade Federal de Santa Maria (UFSM), campus Palmeira das Missões, RS, Brasil. Acta Biológica Catarinense 1: 15-21.

Fiaschi, P. \& Pirani, J.R. 2009. Review of plant biogeographic studies in Brazil. Journal of Systematics and Evolution 47: 477-496.

Filgueiras, T.D.S., Nogueira, P.E., Brochado, A.L. \& Guala, G.F. 1994. Caminhamento: um método expedito para levantamentos florísticos qualitativos. Cadernos de Geociências 12: 39-43.

Flora do Brasil 2020 em construção. Jardim Botânico do Rio de Janeiro. Disponível em: http://floradobrasil.jbrj. gov.br/ (acesso em 14-II-2019).
Forzza, R.C., Baumgratz, J.F.A., Bicudo, C.E.M., Canhos, D.A.L., Carvalho Jr., A.A., Coelho, M.A.N., Costa, A.F., Costa, D.P., Hopkins, M.G., Leitman, P.M., Lohmann, L.G., Lughadha, E.N., Maia, L.C., Martinelli, G., Menezes, M., Morim, M.P., Peixoto, A.L., Pirani, J.R., Prado, J., Queiroz, L.P., Souza, S., Souza, V.C., Stehmann, J.R., Sylvestre, L.S., Walter, B.M.T. \& Zappi, D.V. 2012. New Brazilian Floristic List Highlights Conservation Challenges. BioScience, 62: 39-45.

Gasper, A. L. \& A. Salino. 2016. Samambaias e licófitas de Santa Catarina: composição, riqueza e espécies ameaçadas. Iheringia Série Botânica 70: 321-342.

Gonzatti, F., Machado, L.S. \& Windisch, P.G. 2016. Distribution patterns of ferns and lycophytes in the Coastal Region of the state of Rio Grande do Sul, Brazil. Acta Botanica Brasilica 30: 239-253.

Gonzatti, F., Valduga, E., Wasum, R. A. \& Scur, L. 2014. Florística e aspectos ecológicos de samambaias e licófitas em remanescentes de matas estacionais deciduais da Serra Gaúcha, Rio Grande do Sul, Brasil. Revista Brasileira de Biociências 12: 90-97.

IBGE. 2012. Manual Técnico da Vegetação Brasileira. 2a ed. Rio de Janeiro: IBGE. Disponível em https:// biblioteca.ibge.gov.br/visualizacao/livros/liv63011.pdf (acesso em 14-VI-2019).

Kluge, J. \& Kessler, M. 2010. Phylogenetic diversity, trait diversity and niches: species assembly of ferns along a tropical elevational gradient. Journal of Biogeography 56: 1-10.

Kornás, J. 1977. Life-forms and seasonal patterns in the pteridophytes in Zâmbia. Acta Societatis Botanicorum Poloniae, Warsaw, 46: 669-690.

Kornás, J. 1985. Adaptative strategies of African pteridophytes to extreme environments. In: Dyer, A. F. \& Page, C. N. (eds.) Biology of Pteridophytes. The Royal Society of Edinburg, Edinburg, pp. 391-396.

Lautert, M., Temponi, L.G, Viveros. R.S. \& A. Salino. 2015. Lycophytes and ferns composition of Atlantic Forest conservation units in western Paraná with comparisons to other areas in southern Brazil. Acta Botanica Brasilica 29: 499-508.

Lehn, C.R., Arana, M.D., Bueno, M.L. \& Bianchini, E. 2018. A floristic survey of ferns and lycophytes associated with semi-deciduous remnants in southern Brazil. Darwiniana 6: 133-143.

Lehn, C.R., Leuchtenberger, C. \& Hansen, M.A. 2009. Pteridófitas ocorrentes em dois remanescentes de Floresta Estacional Decidual no Vale do Taquari, Estado do Rio Grande do Sul, Brasil. Iheringia, série botânica 64: 23-31.

Machado, L., Gonzatti, F. \& Windisch, P.G. 2016. Epiphytic ferns in swamp forests of the coastal plain of Southern Brazil: latitudinal effects on plant community. Acta Botanica Brasilica 30: 644-657. 
MapBiomas. 2018. MapBiomas. Disponível em: www. mapbiomas.org (acesso em 14-VI-2019).

Márquez, G.J., Giudice, G.E. \& Ponce, M.M. 2006. Pteridofitas de la Reserva "Valle del Arroyo Cuñá Pirú" (Misiones, Argentina). Darwiniana 44: 108-126.

Mondin, C.A. 2007. Novos registros de Heliantheae Cass. (Asteraceae) para o Brasil. Acta Botanica Brasilica, 21: 993-1001.

Moraes, G.P., Marques, M.W., Bueno, M.L. \& Lehn, C.R. 2018. Samambaias e licófitas da sub-bacia do Rio Fiúza, noroeste do Rio Grande do Sul, Brasil. Pesquisas, botânica 71: 97-107.

Moraes, G.P. \& Lehn, C.R. 2019. Levantamento florístico das samambaias e licófitas do Parque Municipal Rdolfo Arno Goldhart, Panambi, Rio Grande do Sul. Revista de Ciências Ambientais 13: 17-29.

Moura, N.V. 1991. Mapeamento geomorfológico e ambiental do sudoeste do Rio Grande do Sul: Alegrete. Boletim Gaúcho de Geografia 18: 32-42.

Mueller-Dombois, D. \& Ellenberg, H. 1974. Aims and methods of vegetation ecology. New York: John Wiley $\&$ Sons.

Oliveira, E.V. \& Kerber, L. 2009. Paleontologia e aspectos geológicos das sucessões do final do Neógeno no sudoeste do Rio Grande do Sul, Brasil. Gaea 5: 21-34.

Overbeck, G.E., Müller, S.C., Fidelis. A., Pfadenhauer, J., Pillar, V.D., Blanco, C.C., Boldrini, I.I., Both, R. \& Forneck, E.D. 2007. Brazil's neglected biome: the South Brazilian Campos. Perspectives in Plant Ecology, Evolution and Systematics 9: 101-116.

Padoin, T.O.H., Graef, F.V., Silva, V.L. \& Schmitt, J.L. 2015. Florística e aspectos ecológicos das samambaias e licófitas da mata ciliar de um afluente do Rio Rolante no sul do Brasil. Pesquisas, botânica 68: 335-348.

Peel, M.C., Finlayson, B.L. \& McMahon, T.A. 2007. Updated world map of the Koppen-Geiger climate classification. Hydrology and Earth System Science 11: 1633-1644.

Ponce, M.M. \& Scataglini, M.A. 2018. Further progress towards the delimitation of Cheilanthes (Cheilanthoideae, Pteridaceae), with emphasis on South American species. Organisms Diversity \& Evolution 18: 175-186.

Porembski, S. \& Barthlott, W. 2000. Granitic and gneissic outcrops (inselbergs) as centers of diversity for desiccation-tolerant vascular plants. Plant Ecology 151: 19-28.

Porembski, S. 2007. Tropical inselbergs: habitat types, adaptive strategies and diversity patterns. Revista Brasileira de Botânica, 30: 579-586.

PPG I. 2016. A community-derived classification for extant lycophytes and ferns. Journal of Systematics and Evolution 54: 563-603.
Prado, J. \& Hirai, R.Y. 2014. Biogeography of the brazilian atlantic forest: evidence from phylogenetic data sets and perspectives for fern and lycophytes studies. Fern Gazette 19: 241-257

Prado, J. \& Sylvestre, L. 2010. In:R.C.Forzzaetal (org.). Catálogo de plantas e fungos do Brasil. v. 1. Instituto de Pesquisas Jardim Botânico do Rio de Janeiro, Rio de Janeiro, pp. 69-75.

Prado, J., Sylvestre, L., Labiak, P.H., Windisch, P.G., Salino, A., Barros, I.C.L., Hirai, R.Y., Almeida, T.E., Santiago, A.C.P., Kieling-Rubio, M.A., Pereira, A.F.N., Øllgaard, B., Ramos, C.R.V., Mickel, J.T., Dittrich, V.A.O., Mynssen, C.M., Schwartsburd, P.B., Condack, J.P.S., Pereira, J.B.S. \& Matos, F.B. 2015. Diversity of ferns and lycophytes in Brazil. Rodriguésia 66: 1-12.

Roesch, L.F.W., Vieira, F.C.B., Pereira, V.A., Schünemann, A.S., Teixeira, E.F., Senna, A.J.T. \& Stefenon, V.M. 2009. The Brazilian Pampa: A Fragile Biome. Diversity 1: 182-198.

Rossetto, E.F.S. \& Vieira, A.O.S. 2013. Vascular Flora of the Mata dos Godoy State Park, Londrina, Paraná, Brazil. Check List 9: 1020-1034.

Santos, A.C.C. \& Windisch, P.G. 2008. Análise da pteridoflora da área de proteção ambiental do Morro da Borússia (Osório-RS). Pesquisas, botânica 59: 237-252.

Scherer, C.M.S., Faccini, U.F. \& Lavina, E.L. 2000. Arcabouço estratigráfico do Mesozóico da Bacia do Paraná. In: M. Holz \& L.F. Ros (eds.). Geologia do Rio Grande do Sul, Edições CIGO/UFRGS, pp. 335-354.

Schmitt, J.L., Fleck, R., Burmeister, E.L. \& KielingRubio, M.A. 2006. Diversidade e formas biológicas de pteridófitas da Floresta Nacional de Canela, Rio Grande do Sul: contribuições para o plano de manejo. Pesquisas, botânica 57: 275-288.

Sehnem, A. 1970. Polipodiáceas. In: R. Reitz (ed.). Flora Ilustrada Catarinense. Herbário Barbosa Rodrigues, Itajaí, pp. 1-85.

Sehnem, A. 1977. As filicíneas do Sul do Brasil, sua distribuição geográfica, sua ecologia e suas rotas de imigração. Pesquisas, botânica 31: 1-108.

Senna, R.M. \& Waechter, J.L. 1997. Pteridófitas de uma Floresta com Araucária. Formas biológicas e padrões de distribuição geográfica. Iheringia, série botânica 48: 41-58.

Steffens, C. \& Windisch, P.G. 2007. Diversidade e formas de vida de pteridófitas no Morro da Harmonia em Teutônia-RS, Brasil. Pesquisas, botânica 58: 375-382.

Thiers, B. 2019 [continuously updated, accessed 2019]. Index Herbariorum: A global directory of public herbaria and associated staff. New York Botanical Garden's Virtual Herbarium. 
Torres, E.I.M., Sota, E.R. \& Ferrucci, M.S. 2013. Sinopsis de los helechos y licofitos del Parque Nacional Mburucuyá (Corrientes, Argentina): claves de especies. Boletín de la Sociedad Argentina de Botánica 48: 121-136.

Tryon, R.M. \& Tryon, A.F. 1982. Ferns and allied plants with special reference to Tropical America. Springer Verlag, New York.

Verdum R., Vieira L.F.S., Caneppele J.C.G., Gass S.L.B. 2019. Pampa: The South Brazil. In: A. Salgado, L. Santos \& J. Paisani (eds.). The Physical Geography of Brazil. Geography of the Physical Environment. Springer, pp. 7-20.
Welker, C.A.D. \& Longhi-Wagner, H.M. 2012. New records in Schizachyrium (Poaceae, Andropogoneae) for Rio Grande do Sul and for Brazil. Rodriguésia 63: 1147-1150.

Windisch, P.G. 1992. Pteridófitas da região norte-ocidental do Estado de São Paulo: guia para estudo e excursões. 2 ed. Editora UNESP, São José do Rio Preto.

Zona, S. \& Christenhusz, M.J.M. 2015. Litter-trapping plants: filter-feeders of the plant kingdom. Botanical Journal of the Linnean Society 179: 554-586. 